American Journal of Immunology 8 (1): 10-17, 2012

ISSN 1553-619X

(C) 2012 Science Publications

\title{
Maternal Infection and Neurodevelopmental Disorders in the Offspring
}

\author{
Heping Zhou \\ Department of Biological Sciences, Seton Hall University, \\ 400 South Orange Avenue, South Orange, New Jersey 07079, USA
}

\begin{abstract}
Problem statement: Neurodevelopmental disorders such as schizophrenia and autism have been attributed to both genetic and environmental factors. Whether and how maternal infection as an environmental factor contributes to the development of neurological abnormalities in the offspring remains to be clearly defined. Approach: The literature was reviewed to examine the relationship between maternal infection and neurological disorders such as schizophrenia and autism. Results: Both epidemiological and experimental animal studies had found strong support for maternal infection as a significant risk factor for neurodevelopmental disorders. There was also accumulating evidence that inflammatory cytokines and glucocorticoids might be important mediators of maternal infectioninduced effects on the offspring. Other factors such as oxidative stress and hypoxia might also aggravate neurodevelopmental damages. Conclusion: Studies are accumulating to support the link between maternal infection and neurodevelopmental disorders. Mechanisms underlying the link are also unfolding. Future studies examining how maternal infection contributes to the development of different neurodevelopmental disorders can help in developing effective intervention strategies.
\end{abstract}

Key words: Neurodevelopmental disorder, maternal infection, virus, bacteria, neuroinflammation, glucocorticoids, Herpes Simplex Virus (HSV), Gestational Days (GDs)

\section{INTRODUCTION}

Pregnant women have been reported to experience virus infections, such as influenza (Irving et al., 2000), parasitic infections, such as filariasis (Malhotra et al., 2003), gastric infections, such as Helicobacter pylori (Kitagawa et al., 2001), periodontal diseases (gingivitis and periodontitis) (Boutigny et al., 2005; Boggess, 2005; Goepfert et al., 2004) and reproductive infections, such as bacterial vaginosis (Cottrell and Shannahan, 2004). Many studies have reported that maternal infections can have serious and diverse consequences on the health of the fetus, including spontaneous abortions, preterm birth, intrauterine growth retardation, innate immune and neuroimmune functions and other developmentrelated functional outcomes in the offspring (Pararas et al., 2006; Rasmussen and Hayes, 2005; Harry et al., 2006; Brown et al., 2004; Vidaeff and Ramin, 2006; Andrews et al., 2000; Lasala and Zhou, 2007; Hodyl et al., 2008). In fact, more than $70 \%$ of the placental tissues from newborn infants with systemic illness and poor neonatal outcome have positive test results for viral or bacterial infections (Genen et al., 2004; Satosar et al., 2004). There is increasing evidence that maternal infection is a major risk factor for neurodevelopmental brain damages (Chen et al., 2006;
Ashdown et al., 2006; Golan et al., 2005). The objective of this review is to examine the relationship between maternal infection and neurological abnormalities during the later life of the offspring.

Maternal infection and schizophrenia: Schizophrenia is a complex brain disorder involving alterations in cognition, perception, emotion and behavior. At the psychopathological level, schizophrenic subjects often demonstrate deficits in Pre-Pulse Inhibition of startle (PPI) and endogenous sensitization such as increased amphetamine-induced dopamine release (Braff et al., 2001; Laruelle, 2000). The etiology of schizophrenia has been attributed to combinations of genetic and environmental factors. It has been reported that there is a 5-8\% increased risk of schizophrenia among those born in the winter-spring months when infectious diseases are more prevalent and at times when other infections (measles, varicella and poliomyelitis) show increased activity (Yolken, 2004). Epidemiological studies have also found strong association between the development of schizophrenia at later life and maternal infections with viruses such as rubella, influenza and Herpes Simplex Virus (HSV)-2, bacteria such as diphtheria and pneumonia and parasites such as toxoplasmosis (Brown and Susser, 2002; Bresnahan et 
al., 2005; Babulas et al., 2006; Limosin et al., 2003; Ebert and Kotler, 2005; Tamer et al., 2008; Buka et al., 2008; Mortensen et al., 2010).

Many experimental studies using animal models have directly tested if maternal infection results in psychosis-related abnormalities in brain and behavior relevant to schizophrenia in later life. Bacterial products such as lipopolysaccharide (LPS) or viral mimic polyinosinic:polycytidylic acid (poly I:C) have been commonly used to treat pregnant animals to model maternal infection. When LPS is subcutaneously administered to pregnant rats on alternate days during pregnancy, the offspring exhibit deficits in PPI at adulthood (Borrell et al., 2002). Intraperitoneal administration of LPS into pregnant rats on Gestational Days (GDs) 18 and 19 leads to enhanced locomotor response to amphetamine in the adult offspring (Fortier et al., 2004). Intranasal administration of influenza virus on GD 9 causes abnormal corticogenesis in adult mice (Fatemi et al., 2002). The offspring born to poly I: C-treated rats display impaired object recognition memory, loss of latent inhibition and increased sensitivity to the locomotor-stimulating effects of MK801, a non-competitive antagonist of the N-Methyl-DAspartate (NMDA) receptor (Wolff et al., 2011; Zuckerman and Weiner, 2005). Taken together, both epidemiological and experimental animal studies suggest that maternal infection represents an important environmental risk factor for the development of schizophrenia in the offspring at later life.

\section{Maternal infection and Autism Spectrum Disorders (ASDs): ASDs are another group of} neurodevelopmental disorders with both genetic and environmental etiological factors (Rapin and Tuchman, 2008; Johnson and Myers, 2007). The hallmark symptoms of ASDs include deficits in social interaction and language and the presence of repetitive/stereotyped behaviors. Although the causes of ASDs remain to be defined, several areas of studies support maternal infection as a risk factor for ASDs. A link between autism and congenital rubella infection has been suggested by Chess in early 1970s (Chess, 1971) and Ivarsson et al. (1990) reported two cases of autistic children with congenital cytomegalovirus infections in Ivarsson et al. (1990). More recent epidemiological analyses have also shown that maternal infection is associated with diagnosis of ASDs in the offspring (Brown et al., 2008; Atladottir et al., 2010).

The animal models have also provided important insight into the relationship between maternal infection and pathogenesis of ASDs in later life of the offspring. Male offspring mice born to dams treated with poly I:C on GD 10.5 exhibit deficient social and communicative behavior as well as high levels of repetitive behaviors (Malkova et al., 2012). Female rat pups born to dams treated with LPS on GDs 15 and 16 exhibits impaired nest-seeking behavior and odor-stroke associative learning, suggesting that maternal infection affects the social/communicative behavior in the neonate offspring (Baharnoori et al., 2010). Taken together, the animals born to dams treated with LPS or poly I:C exhibit symptoms relevant to autism, suggesting that maternal infection may contribute to the development of ASDs in later life.

Besides schizophrenia and ASDs, Cerebral Palsy (CP), a group of permanent disorders of the development of movement and posture, has also been linked with maternal infection by many epidemiological and experimental studies (Zhou, 2008). It is still unclear how the same insult could contribute to various neurodevelopmental abnormalities. There is some evidence suggesting that the stage of brain development during which the insult is inflicted may play an important role. For example, epidemiological studies by Sorensen et al. (2009) have found that the effects of maternal bacterial infection on offspring risk of ICD-8 schizophrenia is somewhat stronger with an earlier stage of pregnancy (Sorensen et al., 2009). Furthermore, challenge of poly I:C on GD 9 suppresses spatial exploration, impairs sensorimotor gating, reduces prefrontal dopamine D1 receptors and leads to marked enlargement of lateral ventricles in adult mice offspring whereas the same treatment conducted on GD 17 leads to perseverative behavior, impaired working memory, potentiated locomotor reaction to the NMDA-receptor antagonist dizocilpine, reduced hippocampal NMDAreceptor subunit 1 expression and expanded volume of the 4th ventricle without disrupting sensorimotor gating, suggesting that the timing of maternal immune challenge plays a critical role in determining the patterns of behavioral abnormalities offspring display at a later life (Meyer et al., 2006; 2008).

Therefore, maternal infection during different stages of fetal development may lead to distinct brain and behavioral pathologies in adulthood. Further examination and evaluation of maternal immune challenge at different periods of gestation may provide important new insight into the neuroimmunological and neurodevelopmental mechanisms by which different neurological abnormalities arise. Besides the precise timing during pregnancy, the differing neurodevelopmental vulnerabilities and abnormalities following maternal infection may also be associated with the virulence, strain and severity of infection as well as the insults that occur in later life. 
Mechanisms of maternal infection-induced neurodevelopmental disorders: How does maternal infection lead to neurodevelopmental disorders in the offspring? There is still no definitive answer to this question. However, it is known that normal brain development involves precisely timed cellular and molecular events including proliferation, migration, differentiation, myelination, synaptogenesis and elimination of cells and synapses. Disruption of any of these processes could potentially lead to neurotoxicity and long-term brain damage. A common pathway has been suggested to be shared by infecting pathogens considering the diversity of infectious agents during pregnancy that have been linked with neurodevelopmental disorders and inflammatory cytokines and glucocorticoids are among the suggested mediators.

Inflammatory cytokines as a mediator: There are several lines of evidence that support the neuroinflammatory hypothesis. Firstly, as important mediators of immune response, cytokines such as IL$1 \beta$, IL- 6 and TNF- $\alpha$ are induced in maternal and/or fetal tissues during maternal infection (Ashdown et al., 2006; Beloosesky et al., 2006; Burton et al., 2012; Oskvig et al., 2012) and these cytokines are also involved in various aspects of brain development including lineage commitment, proliferation, survival and differentiation of both neuronal and glial cells (Marx et al., 2001; Gilmore et al., 2004; Yang et al., 2002; Mehler and Kessler, 1997; Wang et al., 2002). These cytokines may potentially cross placenta and affect fetal brain development during maternal infection. Consistently, administration of IL-2 into pregnant mice significantly influences the immunological profiles and neurobehavioral patterns in the offspring (Ponzio et al., 2007). Transgenic mice overexpressing IL-6 orTNF- $\alpha$ in the brain also develop severe neurologic diseases (Wang et al., 2002).

Secondly, administration of anti-inflammatory cytokines is able to attenuate maternal infectioninduced toxicity. For example, coadministration of IL-1 receptor antagonist (IL-1ra) alleviates maternal LPSinduced placental inflammation, fetal mortality and motor behavioral alterations in the offspring rats (Girard et al., 2010). NF- $\mathrm{NB}$ inhibitor pyrrolidine dithiocarbamate (PDTC) suppresses the cytokine increases in the early pregnant rats induced by poly I:C administration and reduces the severity of neurodevelopmental defects in adult offspring (Song et al., 2011). Pretreatment with the Nonsteroidal AntiInflammatory Drug (NSAID) Carprofen, a Cyclooxygenase (COX) inhibitor, abrogates maternal poly I:C induced inhibition of neuronal stem cell proliferation (Miranda et al., 2010). Treatment with IL10 , an anti-inflammatory cytokine, significantly reduces the extent of maternal infection-induced fetal brain damage (Rodts-Palenik et al., 2004; Pang et al., 2005).

Thirdly, case studies have shown a strong association between fetal exposure to elevated maternal IL-8 and structural neuroanatomic alterations in schizophrenic patients, suggesting that in utero exposure to elevated IL-8 may partially account for brain disturbances commonly found in schizophrenia (Ellman et al., 2010). Furthermore, an increased maternal level of TNF- $\alpha$ in late gestation period of pregnancy has been associated with cases of psychosis including schizophrenia compared to pregnancies leading to healthy offspring (Buka et al., 2001). Taken together, these findings suggest that cytokines may play a role in the maternal infection-associated neurodevelopmental disorders.

Glucocorticoids as a mediator: Many bacterial and viral infections are known to activate the Hypothalamic-Pituitary-Adrenal (HPA) axis, which ultimately leads to elevated circulating levels of Glucocorticoids (GCs), anti-inflammatory hormones that play a crucial role in restraining and shaping immune responses (Webster and Sternberg, 2004; Bailey et al., 2003; Beishuizen and Thijs, 2003). Under normal conditions, fetal exposure to maternal glucocorticoids is maintained at a low level (Benediktsson et al., 1997). However, the elevated maternal blood level of glucocorticoids induced by maternal infection could expose the fetus to high levels of glucocorticoids, which may influence the offspring's neurodevelopment.

This hypothesis has been tested in experimental animal models. Maternal LPS treatment has been reported to elevate the basal plasma corticosterone level, decrease level of glucocorticoid receptor in the hippocampus of adult offspring rats which exhibit a deficit in Prepulse Inhibition (PPI) and enhanced amphetamine-induced locomotor activity (Reul et al., 1994; Basta-Kaim et al., 2011). Offspring born to pregnant rats treated with dexamethasone on GDs 6-8 exhibit decreased juvenile social play and a blunted acoustic startle reflex in adolescence and adulthood (Kleinhaus et al., 2010). Male offspring mice exposed to stress early in gestation display increased sensitivity to selective serotonin reuptake inhibitor treatment and increased HPA axis responsivity (Mueller and Bale, 2008). Stressful manipulations during the third week of pregnancy have also been shown to reprogram the HPA response in young and adult offspring rats (Henry et al., 1994; Koehl et al., 1999; Ward et al., 2000; Koenig et al., 2005). However, one study by Hauser et al. (2006) reported that male rats prenatally exposed to dexamethasone between GDs 15 and 21 exhibit increased PPI in one of the two replications with 
normal latent inhibition (Hauser et al., 2006 Cambonie et al., 2004). It remains to be determined whether the disparate observations in separate studies could be accounted for by the differences in treatment regimen. Overall, increased fetal exposure to glucocorticoids during maternal infection may contribute to altered neurdevelopmental disorders in the offspring.

It should be noted that other mechanisms may also aggravate maternal infection-induced neurodevelopmental damages in the offspring. For example, bacterial or viral infection-induced oxidative stress may affect neurodevelopment in the fetus (Schwarz, 1996; Cambonie et al., 2004; Lante et al., 2007; Saito et al. 1997). Consistently, post-treatment with NAcetylcysteine (NAC), an antioxidant, after the maternal LPS challenge is able to prevent the drop in the glutathione content in hippocampus and protect LongTerm Potentiation (LTP) in CA1 area of the hippocampus in male offspring (Lante et al., 2008). Infection-induced maternal hypoxia may also impact fetal brain development since maternal hypoxia may lead to fetal hypoxia (Dalitz et al., 2003). Aberrant expressions of neurotrophic factors such as Brain-Derived Neurotrophic Factor (BDNF) and Nerve Growth Factor (NGF) in the fetal brain during maternal infection may also affect the development of the offspring's brain (Golan et al., 2005; Gilmore et al., 2003).

\section{CONCLUSION}

In this review, we examined the relationship between maternal infection and neurodevelopmental disorders in the offspring. A growing body of evidence suggests that inflammatory cytokines and glucocorticoids produced during maternal infection may be primarily responsible for the neurodevelopmental effects that maternal infection has on the offspring. Gestational timing is an important factor to determine the susceptibility and extent of injury of the immature brain to maternal infection. The genetic vulnerabilities of the host and the virulence, strain and severity of infection may also play a role. In addition, the effects of maternal infection may interact with insults that occur in later life to account for the development of neurological symptoms.

Despite the fact that much progress has been made over the years, there are still many questions to be answered. For example, what is the extent of severity and duration of maternal infection that would lead to developmental damage in the fetus? What are the exact mechanisms by which cytokines and/or glucocorticoids affect neurodevelopment? How do cytokines and/or glucocorticoids produce differing effects at different gestational windows? Furthermore, much research is clearly needed to be able to translate results obtained from animal model studies to human development. It will also be of significant benefits if a panel of inflammatory markers in maternal or fetal tissues could be used as a profile to predict neurodevelopmental defects at a later life. Future research addressing these issues may help in developing effective new anti-inflammatory interventions and clinical decision-making.

\section{REFERENCES}

Andrews, W.W., J.C. Hauth and R.L. Goldenberg, 2000. Infection and preterm birth. Am. J. Perinatol.,17: 357-365. PMID: 12141522

Ashdown, H., Y. Dumont, M. Ng, S. Poole and P. Boksa et al., 2006. The role of cytokines in mediating effects of prenatal infection on the fetus: implications for schizophrenia. Molec. Psyc., 11: 47-55. PMID: 16189509

Atladottir, H.O., P. Thorsen, L. Ostergaard, D.E. Schendel and S. Lemcke et al., 2010. Maternal infection requiring hospitalization during pregnancy and autism spectrum disorders. J. Autism Dev. Disor., 40: 1423-1430. PMID: 20414802

Babulas, V., P. Factor-Litvak, R. Goetz, C.A. Schaefer and A.S. Brown, 2006. Prenatal exposure to maternal genital and reproductive infections and adult schizophrenia. Am. J. Psyc., 163: 927-929. PMID: 16648337

Baharnoori, M., S.K. Bhardwaj and L.K. Srivastava, 2010. Neonatal Behavioral Changes in Rats With Gestational Exposure to Lipopolysaccharide: A Prenatal Infection Model for Developmental Neuropsychiatric Disorders. Schizophr. Bull. PMID: 20805287

Bailey, M., H. Engler, J. Hunzeker and J.F. Sheridan, 2003. The hypothalamic-pituitary-adrenal axis and viral infection. Viral Immunol., 16: 141-157. PMID: 12828866

Basta-Kaim, A., B. Budziszewska, M. Leskiewicz, K. Fijal and M. Regulska et al., 2011. Hyperactivity of the hypothalamus-pituitary-adrenal axis in lipopolysaccharide-induced neurodevelopmental model of schizophrenia in rats: Effects of antipsychotic drugs. Eur. J. Pharmacol., 650: 586595. PMID: 21034739

Beishuizen, A. and L.G. Thijs, 2003. Endotoxin and the hypothalamo-pituitary-adrenal (HPA) axis. J. Endotoxin. Res., 9: 3-24. PMID: 12691614

Beloosesky, R., D.A. Gayle, F. Amidi, S.E. Nunez and J. Babu et al., 2006. N-acetyl-cysteine suppresses amniotic fluid and placenta inflammatory cytokine responses to lipopolysaccharide in rats. Am. J. Obstet. Gynecol., 194: 268-273. PMID: 16389042 
Am. J. Immunol., 8 (1): 10-17, 2012

Benediktsson, R., A.A. Calder, C.R. Edwards and J.R. Seckl, 1997. Placental 11 beta-hydroxysteroid dehydrogenase: A key regulator of fetal glucocorticoid exposure. Clin. Endocrinol. (Oxf), 46: 161-166. PMID: 9135697

Boggess, K.A., 2005. Pathophysiology of preterm birth: Emerging concepts of maternal infection. Clin. Perinatol., 32: 561-569. PMID: 16085020

Borrell, J., J.M. Vela, A. Arevalo-Martin, E. MolinaHolgado and C. Guaza, 2002. Prenatal immune challenge disrupts sensorimotor gating in adult rats. Implications for the etiopathogenesis of schizophrenia. Neuropsychopharmacology, 26: 204-215. PMID: 11790516

Boutigny, H., F. Boschin and E. Delcourt-Debruyne, 2005. Periodontal diseases, tobacco and pregnancy. J. Gynecol. Obstet. Biol. Reprod. (Paris), 34: 3S74-83. PMID: 15980775

Braff, D.L., M.A. Geyer and N.R. Swerdlow, 2001. Human studies of prepulse inhibition of startle: normal subjects, patient groups and pharmacological studies. Psychopharmacology, 156: 234-258. PMID: 11549226.

Bresnahan, M., C.A. Schaefer, A.S. Brown and E.S. Susser, 2005. Prenatal determinants of schizophrenia: What we have learned thus far? Epidemiol. Psichiatr. Soc., 14: 194-197. PMID: 16396427

Brown, A.S. and E.S. Susser, 2002. In utero infection and adult schizophrenia. Ment. Retard Dev. Disabil. Res. Rev., 8: 51-57. PMID: 11921387

Brown, A.S., M.D. Begg, S. Gravenstein, C.A. Schaefer and R.J. Wyatt et al., 2004. Serologic evidence of prenatal influenza in the etiology of schizophrenia. Arch. Gen. Psychiatry, 61: 774-780. PMID: 15289276

Brown, G.E., S.D. Jones, A.S. Mackewn and E.J. Plank, 2008. Autism as one of several disabilities in two children with congenital cytomegalovirus infection. Psychol. Reports, 102: 273-282. PMID: 18481687

Buka, S.L., M.T. Tsuang, E.F. Torrey, M.A. Klebanoff and D. Bernstein et al., 2001. Maternal infections and subsequent psychosis among offspring. Arch. Gen. Psyc., 58: 1032-1037. PMID: 11695949

Buka, S.L., T.D. Cannon, E.F. Torrey and R.H. Yolken, 2008. Maternal exposure to herpes simplex virus and risk of psychosis among adult offspring. Biol. Psyc., 63: 809-815. PMID: 17981263

Burton, A., O. Kizhner, M.B. Brown and M.R. Peltier, 2012. Effect of experimental genital mycoplasmosis on gene expression in the fetal brain. J. Reproductive Immunol., 93: 9-16. PMID: 22244476
Cambonie, G., H. Hirbec, M. Michaud, J.M. Kamenka and G. Barbanel, 2004. Prenatal infection obliterates glutamate-related protection against free hydroxyl radicals in neonatal rat brain. J. Neurosci. Res.,75:125-132. PMID: 14689455

Chen, Y.H., D.X. Xu, L. Zhao, H. Wang and J.P. Wang et al., 2006. Ascorbic acid protects against lipopolysaccharide-induced intra-uterine fetal death and intra-uterine growth retardation in mice. Toxicology, 217: 39-45. PMID: 16171921

Chess, S., 1971. Autism in children with congenital rubella. J. Autism. Child. Schizophr., 1: 33-47. PMID: 5172438

Cottrell, B.H and M. Shannahan, 2004. Maternal bacterial vaginosis and fetal/infant mortality in eight Florida counties, 1999-2000. Public. Health Nurs., 21: 395-403. PMID: 15363019

Dalitz, P., R. Harding, S.M. Rees and M.L. Cock, 2003. Prolonged reductions in placental blood flow and cerebral oxygen delivery in preterm fetal sheep exposed to endotoxin: Possible factors in white matter injury after acute infection. J. Soc. Gynecol. Investig, 10: 283-290. PMID: 12853089

Ebert, T. and M. Kotler, 2005. Prenatal exposure to influenza and the risk of subsequent development of schizophrenia. Isr. Med. Assoc. J., 7: 35-38. PMID: 15658144

Ellman, L.M., R.F. Deicken, S. Vinogradov, W.S. Kremen and J.H. Poole et al., 2010. Structural brain alterations in schizophrenia following fetal exposure to the inflammatory cytokine interleukin8. Schizophrenia Res., 121: 46-54. PMID: 20553865

Fatemi, S.H., J. Earle, R. Kanodia, D. Kist and E.S. Emamian et al. 2002. 5.Prenatal viral infection leads to pyramidal cell atrophy and macrocephaly in adulthood: Implications for genesis of autism and schizophrenia. Cellu. Mol. Neurobiol., 22: 25-33. PMID: 12064515

Fortier, M.E., R. Joober, G.N. Luheshi and P. Boksa, 2004. Maternal exposure to bacterial endotoxin during pregnancy enhances amphetamine-induced locomotion and startle responses in adult rat offspring. J. Psychiatr. Res., 38: 335-345. PMID: 15003440

Genen, L., G.J. Nuovo, L. Krilov and J.M. Davis, 2004. Correlation of in situ detection of infectious agents in the placenta with neonatal outcome. J. Pediatr., 144: 316-320. 15001934

Gilmore, J.H., L.F. Jarskog and S. Vadlamudi, 2003. Maternal infection regulates BDNF and NGF expression in fetal and neonatal brain and maternal-fetal unit of the rat. J. Neuroimmunol., 138: 49-55. PMID: 12742653 
Gilmore, J.H., J.L. Fredrik, S. Vadlamudi, J. M. Lauder, 2004. Prenatal infection and risk for schizophrenia: IL-1beta, IL-6, and TNFalpha inhibit cortical neuron dendrite development. Neuropsychopharmacology, 29: 1221-1229. PMID: 15085088

Girard, S., L. Tremblay, M. Lepage and G. Sebire, 2010. IL-1 receptor antagonist protects against placental and neurodevelopmental defects induced by maternal inflammation. J. Immunol., 184: 3997-4005. PMID: 20181892

Goepfert, A.R., M.K. Jeffcoat, W.W. Andrews, O. Faye-Petersen and S.P. Cliver et al., 2004. Periodontal disease and upper genital tract inflammation in early spontaneous preterm birth. Obstet. Gynecol., 104: 777-783. PMID: 15458901

Golan, H.M., V. Lev, M. Hallak, Y. Sorokin and M. Huleihel, 2005. Specific neurodevelopmental damage in mice offspring following maternal inflammation during pregnancy. Neuropharmacology, 48: 903-917. PMID: 15829260

Harry, G.J., C. Lawler and S.H. Brunssen, 2006. Maternal infection and white matter toxicity. Neurotoxicology, 27: 658-670. PMID: 16787664

Hauser, J., J. Feldon and C.R. Pryce, 2006. Prenatal dexamethasone exposure, postnatal development and adulthood prepulse inhibition and latent inhibition in Wistar rats. Behav., Brain Res., 175: 51-61. PMID: 16956676

Henry, C., M. Kabbaj, H. Simon, M. Le Moal and S. Maccari, 1994. Prenatal stress increases the hypothalamo-pituitary-adrenal axis response in young and adult rats. J. Neuroendocrinol., 6: 341-345. PMID: 7920600

Hodyl, N.A., K.M. Krivanek, V.L. Clifton and D.M. Hodgson, 2008. Innate immune dysfunction in the neonatal rat following prenatal endotoxin exposure. J. Neuroimmunol., 204: 126-130. PMID: 18708268

Irving, W.L., D.K. James, T. Stephenson, P. Laing and C. Jameson et al., 2000. Influenza virus infection in the second and third trimesters of pregnancy: A clinical and seroepidemiological study. BJOG, 107: 1282-1289. PMID: 11028582

Ivarsson, S.A., I. Bjerre, P. Vegfors and K. Ahlfors, 1990. Autism as one of several disabilities in two children with congenital cytomegalovirus infection. Neuropediatrics, 21: 102-103. PMID: 2163029

Johnson, C.P. and S.M. Myers, 2007. Identification and evaluation of children with autism spectrum disorders. Pediatrics, 120: 1183-1215. PMID: 17967920
Kitagawa, M., M. Natori, M. Katoh, K. Sugimoto and H. Omi et al., 2001. Maternal transmission of helicobacter pylori in the perinatal period. J. Obstet. Gynaecol. Res., 27: 225-230. PMID: 11721735

Kleinhaus, K., S. Steinfeld, J. Balaban, L. Goodman and T.S. Craft et al., 2010. Effects of excessive glucocorticoid receptor stimulation during early gestation on psychomotor and social behavior in the rat. Dev. Psychobiol., 52: 121-132. PMID: 20091810

Koehl, M., M. Darnaudery, J. Dulluc, O. Van Reeth and M. Le Moal et al., 1999. Prenatal stress alters circadian activity of hypothalamo-pituitary-adrenal axis and hippocampal corticosteroid receptors in adult rats of both gender. J. Neurobiol., 40: 302315. PMID: 10440731

Koenig, J.I., G.I. Elmer, P.D. Shepard, P.R. Lee and C. Mayo et al., 2005. Prenatal exposure to a repeated variable stress paradigm elicits behavioral and neuroendocrinological changes in the adult offspring: Potential relevance to schizophrenia. Behav Brain Res., 156: 251-261. PMID: 15582111

Lante, F., J. Meunier, J. Guiramand, T. Maurice and M. Cavalier et al., 2007. Neurodevelopmental damage after prenatal infection: Role of oxidative stress in the fetal brain. Free Radic. Biol. Med., 42: 1231-1245. PMID: 17382204

Lante, F., J. Meunier, J. Guiramand, M.C. De Jesus Ferreira and G. Cambonie et al., 2008. Late Nacetylcysteine treatment prevents the deficits induced in the offspring of dams exposed to an immune stress during gestation. Hippocampus, 18: 602-609. PMID: 18306297

Laruelle, M., 2000. The role of endogenous sensitization in the pathophysiology of schizophrenia: implications from recent brain imaging studies. Brain. Res. Brain Res. Rev., 31: 371-384. PMID: 10719165

Lasala, N. and H. Zhou, 2007. Effects of maternal exposure to LPS on the inflammatory response in the offspring. J. Neuroimmunol., 189: 95-101. PMID: 17706793

Limosin, F., F. Rouillon, C. Payan, J.M. Cohen and N. Strub, 2003. Prenatal exposure to influenza as a risk factor for adult schizophrenia. Acta. Psychiatr. Scand., 107: 331-335. PMID: 12752028

Malhotra, I., J.H. Ouma, A. Wamachi, J. Kioko and P. Mungai et al., 2003. Influence of maternal filariasis on childhood infection and immunity to Wuchereria bancrofti in Kenya. Infect. Immune., 71: 5231-5237. PMID:12933869 
Am. J. Immunol., 8 (1): 10-17, 2012

Malkova, N.V., C.Z. Yu, E.Y. Hsiao, M.J. Moore and P.H. Patterson, 2012. Maternal immune activation yields offspring displaying mouse versions of the three core symptoms of autism. Brain, Behav. Immun. PMID: 22310922

Marx, C.E., L.F. Jarskog, J.M. Lauder, J.A. Lieberman and J.H. Gilmore, 2001. Cytokine effects on cortical neuron MAP-2 immunoreactivity: Implications for schizophrenia. Biol. Psyc., 50: 743-749. PMID: 11720692

Mehler, M.F. and J.A. Kessler, 1997. Hematolymphopoietic and inflammatory cytokines in neural development. Trends Neurosci., 20: 357365. PMID: 9246730

Meyer, U., M. Nyffeler, A. Engler, A. Urwyler and M. Schedlowski et al., 2006. The time of prenatal immune challenge determines the specificity of inflammation-mediated brain and behavioral pathology. J. Neurosci., 26: 4752-4762. PMID: 16672647.

Meyer, U., M. Nyffeler, B.K. Yee, I. Knuesel and J. Feldon, 2008. Adult brain and behavioral pathological markers of prenatal immune challenge during early/middle and late fetal development in mice. Brain. Behav. Immun., 22: 469-486. PMID: 18023140.

Miranda, D.J., K. Yaddanapudi, M. Hornig, G. Villar and R. Serge et al., 2010. Induction of Toll-like receptor 3-mediated immunity during gestation inhibits cortical neurogenesis and causes behavioral disturbances. MBio. PMID: 20941330

Mortensen, P.B., C.B. Pedersen, D.M. Hougaard, B. Norgaard-Petersen and O. Mors et al., 2010. A Danish National Birth Cohort study of maternal HSV-2 antibodies as a risk factor for schizophrenia in their offspring. Schizophrenia Res., 122: 257263. PMID: 20598509

Mueller, B.R. and T.L. Bale, 2008. Sex-specific programming of offspring emotionality after stress early in pregnancy. J. Neurosci., 28: 9055-9065. PMID: 18768700

Oskvig, D.B., A.G. Elkahloun, K.R. Johnson, T.M. Phillips and M. Herkenham, 2012. Maternal immune activation by LPS selectively alters specific gene expression profiles of interneuron migration and oxidative stress in the fetus without triggering a fetal immune response. Brain. Behav. Immun. PMID: 22310921

Pang, Y., S. Rodts-Palenik, Z. Cai, W.A. Bennett and P.G. Rhodes, 2005. Suppression of glial activation is involved in the protection of IL-10 on maternal E. coli induced neonatal white matter injury. Brain. Res. Dev. Brain. Res., 157: 141-149. PMID: 15878785
Pararas, M.V., C.L. Skevaki and D.A. Kafetzis, 2006. Preterm birth due to maternal infection: Causative pathogens and modes of prevention. Eur. J. Clin. Microbiol. Infect. Dis., 25: 562-569. PMID: 16953371

Ponzio, N.M., R. Servatius, K. Beck, A. Marzouk and T. Kreider, 2007. Cytokine levels during pregnancy influence immunological profiles and neurobehavioral patterns of the offspring. Ann. N Y Acad. Sci., 1107: 118-128. PMID: 17804539

Rapin, I. and R.F. Tuchman, 2008. Autism: definition, neurobiology, screening, diagnosis. Pediatr. Clin. North. Am., 55: 1129-1146. PMID: 18929056

Rasmussen, S.A. and E.B. Hayes, 2005. Public health approach to emerging infections among pregnant women. Am. J. Public. Health, 95: 1942-1944. PMID: 16195518

Reul, J.M., I. Stec, G.J. Wiegers, M.S. Labeur and A.C. Linthorst et al. 1994. Prenatal immune challenge alters the hypothalamic-pituitary-adrenocortical axis in adult rats. J. Clin. Invest., 93: 2600-2607. PMID: 8200998

Rodts-Palenik, S., J. Wyatt-Ashmead, Y. Pang, B. Thigpen and Z. Cai et al., 2004. Maternal infection-induced white matter injury is reduced by treatment with interleukin-10. Am. J. obstet. Gynecol., 191: 1387-1392. PMID: 15507970

Saito, K., S. Packianathan and L.D. Longo, 1997. Free radical-induced elevation of ornithine decarboxylase activity in developing rat brain slices. Brain Res., 763: 232-238. PMID: 9296564

Satosar, A., N.C. Ramirez, D. Bartholomew, J. Davis and G.J. Nuovo, 2004. Histologic correlates of viral and bacterial infection of the placenta associated with severe morbidity and mortality in the newborn. Hum. Pathol., 35: 536-545. PMID: 15138926

Schwarz, K.B., 1996. Oxidative stress during viral infection: A review. Free Radic. Biol. Med., 21: 641-649. PMID: 8891667

Song, X., W. Li, Y. Yang, J. Zhao and C. Jiang et al. 2011. The nuclear factor- $\kappa \mathrm{B}$ inhibitor pyrrolidine dithiocarbamate reduces polyinosinic-polycytidilic acid-induced immune response in pregnant rats and the behavioral defects of their adult offspring. Behav., Brain. Funct., 7: 50-50. PMID: 22208616

Sorensen, H.J., E.L. Mortensen, J.M. Reinisch and S.A. Mednick, 2009. Association between prenatal exposure to bacterial infection and risk of schizophrenia. Schizophrenia Bull., 35: 631-637. PMID: 18832344 
Tamer, G.S., D. Dundar, I. Yalug, S. Caliskan and S. Yazar et al., 2008. The schizophrenia and Toxoplasma gondii connection: Infectious, immune or both? Adv. Ther., 25: 703-709. PMID: 18563312

Vidaeff, A.C. and S.M. Ramin, 2006. From concept to practice: the recent history of preterm delivery prevention. Part II: Subclinical infection and hormonal effects. Am. J. Perinatol., 23: 75-84. PMID: 16506112

Wang, J., V.C. Asensio and I.L. Campbell, 2002. Cytokines and chemokines as mediators of protection and injury in the central nervous system assessed in transgenic mice. Curr. Top. Microbiol. Immun., 265: 23-48. PMID: 12014193

Ward, H.E., E.A. Johnson, A.K. Salm and D.L. Birkle, 2000. Effects of prenatal stress on defensive withdrawal behavior and corticotropin releasing factor systems in rat brain. Physiol. Behav., 70: 359-366. PMID: 11006435

Webster, J.I. and E.M. Sternberg, 2004. Role of the hypothalamic-pituitary-adrenalaxis, glucocorticoids and glucocorticoid receptors in toxic sequelae of exposure to bacterial and viral products. J. Endocrinol., 181: 207-221. PMID: 15128270
Wolff, A.R., K.R. Cheyne and D.K. Bilkey, 2011. Behavioural deficits associated with maternal immune activation in the rat model of schizophrenia. Behav. Brain Res., 225: 382-387. PMID: 21816179

Yang, L., K. Lindholm, Y. Konishi, R. Li and Y. Shen, 2002. Target depletion of distinct tumor necrosis factor receptor subtypes reveals hippocampal neuron death and survival through different signal transduction pathways. J Neurosci., 22: 3025-3032. PMID: 11943805

Yolken, R., 2004. Viruses and schizophrenia: A focus on herpes simplex virus. Herpes, 2: 83A-88A. PMID: 15319094

Zhou, H., 2008. Mechanistic links between maternal bacterial infection and cerebral palsy. Biotechnol. Mol. Biol. Rev., 3: 14-23.

Zuckerman, L. and I. Weiner, 2005. Maternal immune activation leads to behavioral and pharmacological changes in the adult offspring. J. Psychiatr. Res., 39: 311-323. PMID: 15725430 